\title{
A Supercut of Supercuts: Aesthetics, Histories, Databases
}

MAX TOHLINE (1)

ब나

Open Library of Humanities

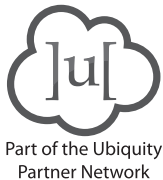

CORRESPONDING AUTHOR:

Max Tohline

Independent scholar, US

maxtohline@gmail.com The genealogies of the supercut, which extend well past YouTube compilations, back
to the 1920 s and beyond, reveal it not as an aesthetic that trickled from avant-garde experimentation into mass entertainment, but rather the material expression of a newly-ascendant mode of knowledge and power: the database episteme.

\section{ABSTRACT} The genealogies of the supercut, which extend well past YouTube compilations, back
to the 1920s and beyond, reveal it not as an aesthetic that trickled from avant-garde 


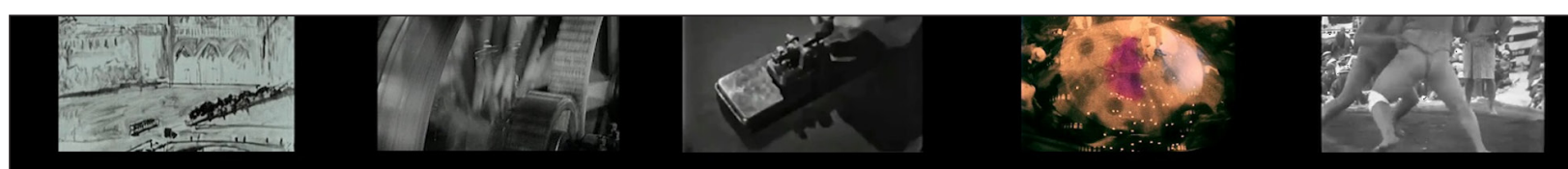

Some shots were cut out to be something else.
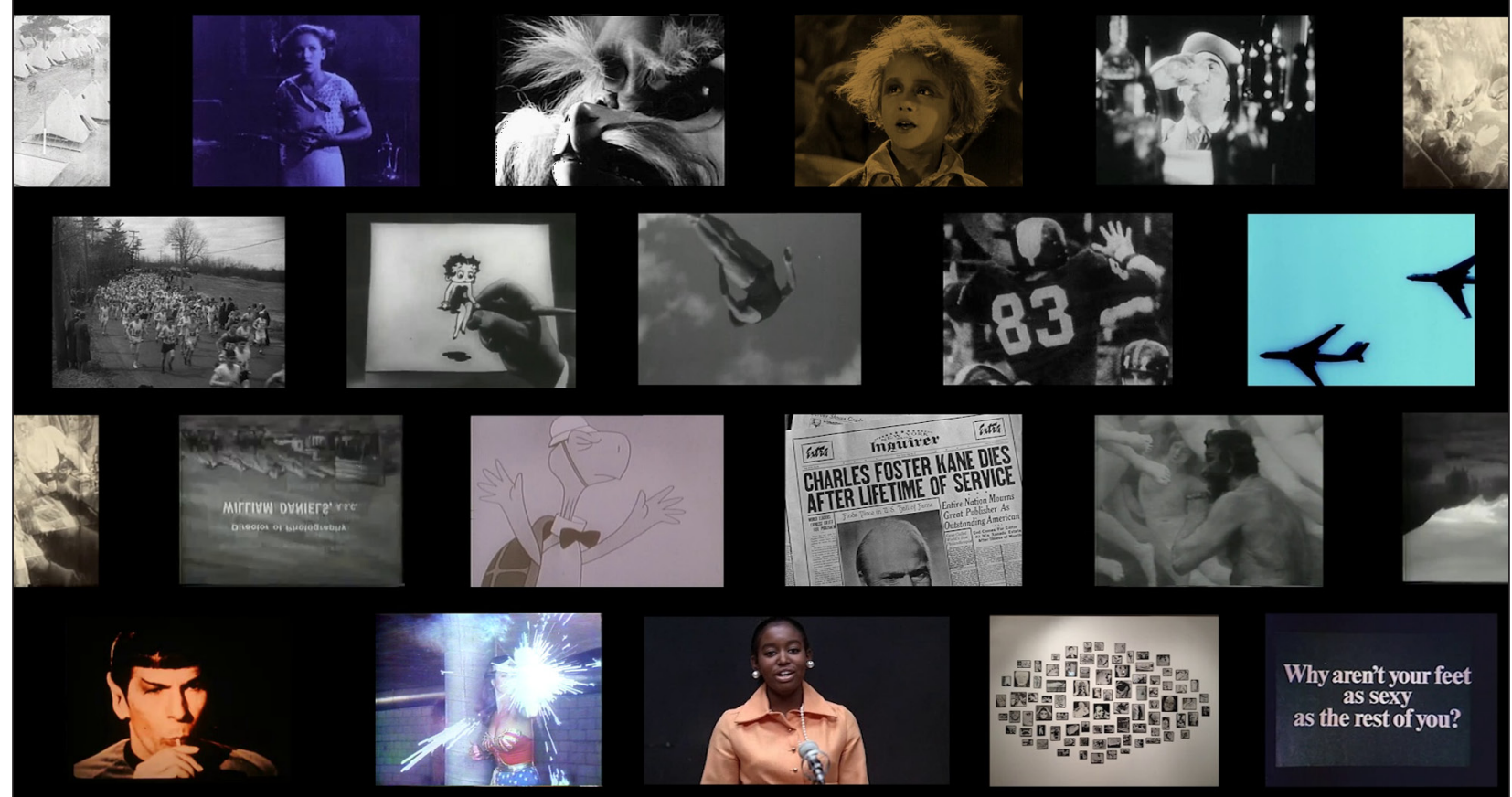

\section{OPEN \\ SCREENS}

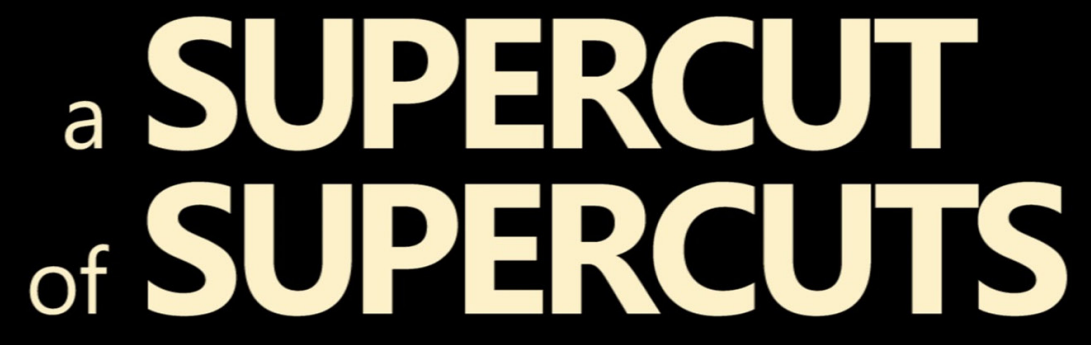

Aesthetics | Histories | Databases
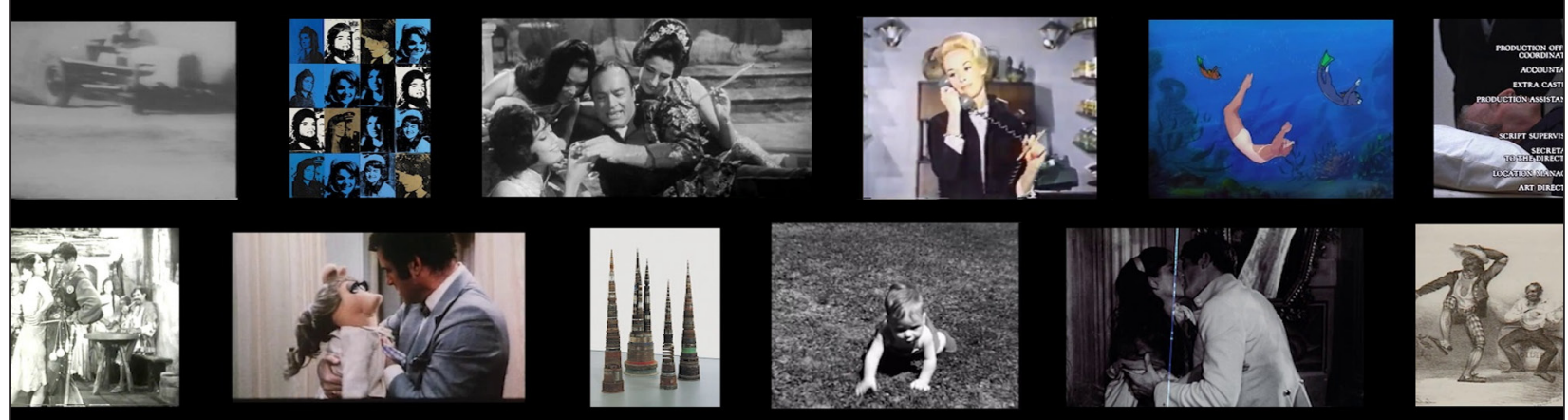

\section{A Video Essay by Max Tohline}

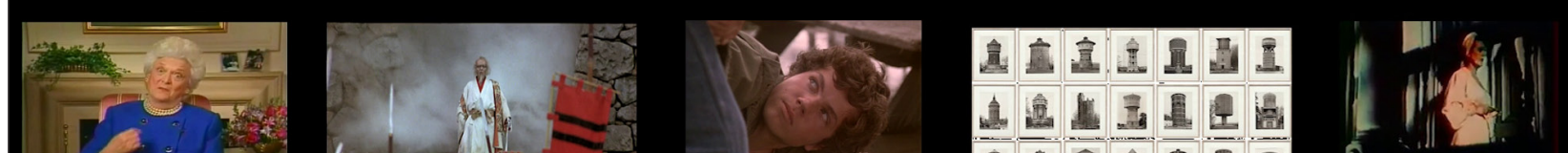

Full Transcript: https://www.academia.edu/45172369/Tohline_A_Supercut_of_Supercuts_full_transcript. 


\section{RESEARCH STATEMENT}

This first inklings of this video essay came in the form of a one-off blog post I wrote seven years ago (Tohline 2013) in response to Miklos Kiss's work on the "narrative" supercut (Kiss 2013). My thoughts then comprised little more than a list; an attempt to add a few works to the prehistory of the supercut that I felt Kiss and other supercut researchers or popularizers, like Tom McCormack (2011) and Andy Baio (2008), had left out. As time went by and the aesthetic of assembling appropriated footage into a matching list of clips spread far beyond fan montages on YouTube, I felt it was time to return to the history of the supercut, try to fill in the gaps of the existing work on it, and account for the social meaning behind its proliferation. And that's how this video essay began: with a hunch that a fad had somehow become ideological. As I performed over a year of image-research and encountered quite a few more cultural tributaries of the supercut in places I had not expected, it became clear that the supercut was never just a subset of remix, compilation, or fan culture, but was instead a material expression of a new historical mode of knowledge and power: the database episteme. The supercut was not invented within the avant-garde, later making its way into more populist forms of entertainment; rather, the roots of the form appeared simultaneously in many places at once. This phenomenon of multiple-emergence is most evident in the 1920s, where montages that first resemble what would later be called supercuts pop up in newsreels, sight gags in silent comedies, city symphonies, agit-prop documentary, German crosssection montages, mainstream narratives, and more (see, for example, The Cruise of the Jasper B. (1926), Rien que les heures (1926), Turksib (1929), Melody of the World (1929), or The Crowd (1928)). Despite the fact that the supercut embodies database-thinking, or at least a kind of simulation of a computer search operation, up to this point our histories of the supercut have been primarily archival, focusing on a few favored works and creators while not noticing the bulk of the tradition. This project thus primarily represents an attempt to bring a more rounded database-approach to the histories of the supercut, a database-form.

Structurally, this essay breaks up into three parts. Part one analyses the aesthetics of the supercut in terms of what kind of attention the form naturally emphasizes or intensifies. Drawing on Tim Smith's Attentional Theory of Cinematic Continuity (Smith 2012) and Eli Horwatt's work on "Instrumental Appropriation" in photocollage (Horwatt 2013), I argue that there is no semiotic or structuralist reason why viewers should prefer narrative patterning to database patterning of images. In fact, just as the conventions of classical continuity editing direct spectatorial attention toward elements of the unfolding narrative and away from extraneous details, so also the strong patterning in supercuts focuses viewer attention toward that which repeats, stoking uncritical desire for that repetition, regardless of the content of the images. While critical analysis is certainly possible within the form, the supercut, broadly speaking, naturally gravitates toward desire instead of analysis.

Armed with this conclusion, part two sets out to discover the various roots of the supercut with this desire-centered-ness, and other pragmatics, as a guide. Deploying over a hundred video clips, I show how existing histories of the supercut, which tend to center on avant-garde filmmaking or fan cultures, both fail to mention a great deal of relevant works and, in some cases, greatly misunderstand the nature of the form. To correct these errors, I produce an original genealogy of the various tributaries of the moving image that feed into the modern supercut, including: the cinephilic thread, which stretches from Cinema Paradiso (1988) and That's Entertainment (1974) back to German and American film history montages of the 1920s; the crosssection thread, which begins with Walter Ruttmann's querschnitt montages of the 1920s and extends through the avant-garde work of later found footage filmmakers; the archival-analysis thread, with its roots in Shub and Eisenstein, that blossoms in the visual culture work of Berger, Loader \& the Raffertys, and others; and the narrative thread, emerging in Vorkapich-style montages of the 1920s and 30s and continuing in later attempts to compress serial narrative events or express a character's essayistic thoughts.

With these various cultural series of the supercut in place, part three theorizes the histories of the supercut as a tale of the emergence of the database episteme. Over the past century, we have come to think of nearly every aspect of our lives as composed of data. The supercut is part of this; indeed, every time we use a search engine, we commission a computer to make us something quite like a supercut. Thus, the supercut entails not simply a mode of editing, but a mode of thinking expressed by a mode of editing. Accordingly, in part three I argue for the supercut as an example of what happens when an episteme takes root ideologically well before the technologies corresponding to the world it envisions have been invented. Just as capitalism treated workers as machines as a prelude to workers being replaced by machines, so also supercutters simulate database thinking in apparent anticipation of a moment, perhaps in the near future, when neural networks will be able to search the entirety of digitized film history and create supercuts themselves, automatically. In its desirecentered aesthetics and its often-evidentiary uses, the supercut carries many of the promises and threats of the database episteme. But like any technology, the supercut's threats need not outweigh its promises. In my conclusion, I imagine decoupling database-thinking from its service to capitalist systems of control and argue that 
now is the time to imagine what this liberation of the database episteme could look like, both in the supercut and across culture more broadly.

\section{COMPETING INTERESTS}

The author has no competing interests to declare.

\section{AUTHOR AFFILIATION}

Max Tohline (D) orcid.org/0000-0002-7065-6473

Independent scholar, US

\section{REFERENCES \\ MAIN BIBLIOGRAPHY}

Ades, D. 2016. The Strangeness of Cornell's Rose Hobart. In: Augaitis, D, et al. (eds.), MashUp: The Birth of Modern Culture. E.U.: Black Dog Publishing Limited, pp. 78-81.

Baio, A. 2008. Fanboy Supercuts, Obsessive Video Montages, 11 April 2008. Available at https://waxy.org/2008/04/ fanboy_supercuts_obsessive_video_montages/ [Accessed 3 December 2020].

Bateman, J. 2007. Towards a grande paradigmatique of film: Christian Metz reloaded. Semiotica, 2007(167): 13-64. DOI: https://doi.org/10.1515/SEM.2007.070

Bazin, A. 1946. The Myth of Total Cinema. In: Bazin, A (ed.), 1967 What is Cinema?, Vol. 1, trans. H. Gray. Berkeley: University of California Press.

Benjamin, W. 1936. The Work of Art in the Age of Its Technological Reproducibility. In: Jennings, M, Doherty, $B$ and Levin, $T$ (eds.), The Work of Art in the Age of Its Technological Reproducibility, and Other Writings on Media, trans. E Jephcott, R Livingstone, H Eiland, et al. Cambridge: Harvard University Press.

Berliner, A. n.d. The Family Album, viewed 13 December 2020, http://www.alanberliner.com/first_cousin.php?pag_id=37.

Bordwell, D, Staiger, J and Thompson, K. 1985. The Classical Hollywood Cinema: Style and Mode of Production to 1960. London: Routledge. DOI: https://doi.org/10.2307/1772109

Coppa, F. 2014. Interview with Kandy Fong. In Rehak, B (ed.) Materiality and Object-Oriented Fandom, special issue of Transformative Works and Cultures, (16). DOI: https://doi. org/10.3983/twc.2014.0535

Cowan, M. 2013. Cutting through the Archive: Querschnitt Montage and Images of the World in Weimar Visual Culture. New German Critique, 40(3). DOI: https://doi. org/10.1215/0094033X-2325410

Deleuze, G. 1986. Cinema 1: the movement-image, trans. H. Tomlinson \& B. Hammerjam. Minneapolis: University of Minnesota Press, pp. 36-37. DOI: https://doi. org/10.5040/9781350251977

Eisenstein, S. 1949. Film Form: Essays in Film Theory, trans. J. Leyda. New York: Harcourt, Inc.

Farhi, P. 2012. A local TV trend: And now, the identical news.
The Washington Post, 27 November 2012. Available at https://www.washingtonpost.com/lifestyle/style/a-local-tvtrend-and-now-the-identical-news/2012/11/27/9369a30838bd-11e2-8a97-363b0f9a0ab3_story.html [Accessed 24 December 2020].

Foucault, M. 1984. What is an Author? In: Rabinow, P (ed.), The Foucault Reader. New York: Pantheon Books.

Foucault, M. 2004. The Order of Things: An Archaeology of Human Sciences. New York: Routledge Classics.

Gallagher, 0. 2012. Remix Semiosis as Ideology Critique: A Visual Semiotic Study of Critical Remix Video. Gramma: Journal of Theory and Criticism, 20: 127-144. DOI: https:// doi.org/10.26262/gramma.v20i0.6298

Hagener, M. 2007. Moving Forward, Looking Back: The European Avant-garde and the Invention of Film Culture, 1919-1939. Amsterdam: Amsterdam University Press. DOI: https://doi. org/10.5117/9789053569610

Horwatt, E. 2013. On The Clock and Christian Marclay's Instrumental Logic of Appropriation. Framework, 54(2): 208-225. DOI: https://doi.org/10.13110/ framework.54.2.0208

Jacobs, S, Kinik, A and Hielscher, E. 2019. The City Symphony Phenomenon: Cinema, Art, and Urban Modernity Between the Wars. New York: Routledge. DOI: https://doi. org/10.4324/9781315619989

Kastrenakes, J. 2019. Fox News stops TV recording services that let journalists search for clips. The Verge, 21 January 2019. Available at https://www.theverge. com/2019/1/21/18191652/fox-news-tveyes-settlementtv-recording-monitoring-service-fair-use [Accessed 23 December 2020].

Kiss, M. 2013. Creativity Beyond Originality: György Pálfi's Final Cut as Narrative Supercut. Senses of Cinema 67. Available at: http://www.sensesofcinema.com/2013/feature-articles/ creativity-beyond-originality-gyorgy-palfis-final-cut-asnarrative-supercut/ [Accessed 23 December 2020].

Kracauer, S. 1947. Montage. In: Kahana, J (ed.), 2016 The Documentary Film Reader: History, Theory, Criticism. New York: Oxford University Press, pp. 142-147.

Leyda, J. 1964. Films Beget Films: A Study of the Compilation Film. New York: Hill and Wang.

Lopez, A, et al. 1997. The Tree of Life: Universal and Cultural Features of Folkbiological Taxonomies and Inductions. Cognitive Psychology, 32: 251-295. DOI: https://doi. org/10.1006/cogp.1997.0651

MacDonald, S. 1988. A Critical Cinema: Interviews with Independent Filmmakers. Berkeley: University of California Press. DOI: https://doi.org/10.1525/9780520939080

MacDonald, S. 2005. A Critical Cinema 4: Interviews with Independent Filmmakers. Berkeley: University of California Press.

Manovich, L. 2001. The Language of New Media. Cambridge, Massachusetts: The MIT Press.

Marx, K. 1858. The Grundrisse. In: Tucker, R (ed.), 1978 The Marx-Engels Reader, $2^{\text {nd }}$ edition. New York: W. W. Norton \& Co. 
McCormack, T. 2011. Compilation Nation: The history and the rise of the supercut. Museum of the Moving Image: Moving Image Source, 25 April 2011. Available at http:// www.movingimagesource.us/articles/compilationnation-20110425 [Accessed 24 December 2020].

Meneghelli, D. 2017. Just Another Kiss: Narrative and Database in Fan Vidding 2.0. Global Media Journal: Australian Edition, 11(1): 1-14.

Metz, C. 1966. La grande syntagmatique du film narratif. Communications, 8(1): 120-124. DOI: https://doi. org/10.3406/comm.1966.1119

Metz, C. 1974. Film Language: A Semiotics of the Cinema. Chicago: University of Chicago Press.

Miller, B [he4ts3eker]. 2016. We Love Video: The Evolution of the Supercut, 18 July 2016. Available at https://brenkjm. wordpress.com/2016/07/18/the-evolution-of-the-supercut/ [Accessed 22 July 2019].

Morris, I. (ed.) 1991. The Pillow Book of Sei Shonagon [ca. 1002], trans. I Morris, New York: Columbia University Press.

Mozur, P. 2018. Inside China's Dystopian Dreams: A.I., Shame and Lots of Cameras. The New York Times, 8 July 2018, Available at https://nytimes.com/2018/07/08/business/ china-surveillance-technology.html [Accessed 24 December 2020].

Murray, B. 2015. Remixing Culture And Why The Art Of The Mash-Up Matters, 22 March 2015. Available at https:// techcrunch.com/2015/03/22/from-artistic-to-technologicalmash-up/ [Accessed 4 December 2020].

PBS. n.d. POV: The Family Album, viewed 13 December 2020, https://www.pbs.org/pov/thefamilyalbum/.

Queneau, R. 1947. Exercises in Style, trans. B Wright (1981). New York: New Directions Books.

Rebick, S. 2016. The Invention of the Supercut. In: Augaitis, D, et al. (eds.), MashUp: The Birth of Modern Culture. Vancouver: Black Dog Publishing Limited, pp. 146-151.

Russell, C. 2018. Archiveology: Walter Benjamin and Archival Film Practices. Durham: Duke University Press. DOI: https:// doi.org/10.1215/9780822372004

Sadowski, J. 2018. Potemkin AI. Real Life Mag, 6 Aug. 2018. Available at: https://reallifemag.com/potemkin-ai/ [Accessed 9 December 2020].

Smith, T. 2012. The Attentional Theory of Cinematic Continuity. Projections, 6(1): 1-27. DOI: https://doi.org/10.3167/ proj.2012.060102

Tohline, M. 2013. Why Not a Supercut of Supercuts? (Tumblr post), 7 December, https://10oclockdot.tumblr.com/ post/69270352197/.

Whitman, W. 1855. There Was a Child Went Forth. In: Whitman, W (ed.), 1871 Leaves of Grass. New York: Smith \& McDougal, Available at https://whitmanarchive.org/published/LG/1871/ poems/113 [Accessed 7 December 2020].

Yumibe, J. 2013. French Film Colorists. In: Gaines, J, Vatsal, R and Dall'Asta, M (eds.), Women Film Pioneers Project. New York: Columbia University Libraries. Available at: https:// wfpp.columbia.edu/essay/french-film-colorists/ [Accessed on 23 December 2020].
Zalewski, D. 2012. The Hours: How Christian Marclay created the ultimate digital mosaic. The New Yorker, March 4, 2012. Online: https://www.newyorker.com/magazine/2012/03/12/ the-hours-daniel-zalewski [Accessed 12 December 2020].

\section{FILM AND VIDEO MATERIAL}

\#1 - Nightclubbing, 2017. [Web video] TROISCOULEURS.

100 Movies Dance Scenes Mashup (Mark Ronson-Uptown Funk

ft.Bruno Mars), 2015. [Web video] What's the Mashup.

100 Movies, 100 Quotes, 100 Numbers, 2007. [Web video]

AlonzoMosleyFBI.

100 Photographs: The Most Influential Images of All Time Trailer, 2017. [Web video] TIME.

19 Waxing Scenes in Film and Television, 2018. [Web video] New York Magazine.

200 Mouths to Feed, 1994 [Web video] Claude Closky.

$20^{\text {th }}$ Century Women, 2016 [Blu-ray] Mike Mills.

269. What Makes a Video Essay Great?, 2014. [Web video] Kevin B. Lee.

3000 Years of Art, 1968. [Web video] Dan McLaughlin.

38 Seconds With Gustavo Almadovar, 2008. [Web video]

AlmodovarGustavo.

500 Years of Female Portraits in Western Art, 2007. [Web video]

Philip Scott Johnson.

555-SUPERCUT, 2011. [Web video] GoSwarmIt.

56 Ways of Saying I Don't Remember, 2013. [Web video] Alan Berliner.

6 Hammers from Mahler's Symphony no. 6 'Tragic', 2014. [Web video] Max Tohline.

60 Seconds, 2003. [Web video] Christoph Girardet.

66 Movie Dance Scenes Mashup with Can't Stop the Feeling by Justin Timberlake, 2016. [Web video] Movie Remixer.

787 Cliparts, 2006. [DVD] Oliver Laric.

80s Computer Hacking: A Supercut, 2014. [Web video]

FoundItemClothing.com.

A Brief Look at Texting and the Internet in Film, 2014. [Web video] Every Frame a Painting [Tony Zhou].

A Crazy Composer, 1905. [DVD] Georges Méliès.

A Damn Good Coffee Tribute, 2016. [Web video] Anna Catley.

A Devastating Tribute to the Single Tear, 2014. [Web video] Nerdwriter1.

A Movie, 1958. [VHS] Bruce Conner.

A Movie by Jen Proctor, 2010. [Web video] Jen Proctor.

A Song from the Cultural Revolution, 1998. [DVD] Les LeVeque.

A Supercut of Supercuts: Aesthetics, Histories, Databases, 2020.

[Digital file] Max Tohline.

A Trip to the Moon, 1902 [Blu-ray] Georges Méliès.

ABC World News Tonight, 23 May 1983. [Web video] American Broadcasting Companies, Inc.

Alfred Butterworth and Sons, Glebe Mills, Hollinwood, 1901. [DVD] Mitchell and Kenyon.

Alfred Hitchcock's 39 Stairs [2 loops], 2017. [Web video] Max Tohline.

All That Jazz, 1979. [Blu-ray] Bob Fosse.

All the times President Trump praised WikiLeaks, 2019. [Web video] CBS News. 
Amélie, 2001. [Blu-ray] Jean-Pierre Jeunet.

American Time Capsule, 1968. [Web video] Chuck Braverman.

America's Funniest Home Videos, Season 5, Episode 2 "Domino

Baby," 1993. [Web video] Steve Hirsen.

An Adventurous Automobile Trip, 1905. [DVD] Georges Méliès.

Analepsis, 2003-2004. [Web video] Sean Snyder.

Apple 'Hello' iPhone Commercial, 2007. [Web video] TBWA/Apple.

ARST ARSW: Star Wars sorted alphabetically, 2014. [Web video]

suckerpinch.

Artist, 1999. [Web video] Tracey Moffatt \& Gary Hillberg.

Asphalt, 1929. [DVD] Joe May.

At Any Price, 2012. [DVD], Ramin Bahrani [screenshot only].

At The Academy, 1974. [DVD] Guy Sherwin.

Auto-H.S.S., 2019. [Web video] Max Tohline.

Babel, 2006. [Poster] Alejandro G. Iñárritu.

Ballet Mécanique, 1924. [DVD] Fernand Léger.

Bamboozled, 2000. [Blu-ray] Spike Lee.

Barack Obama Singing Drink to That by Rihanna [OFFICIAL],

2012. [Web video] baracksdubs.

Becoming, 2020. [Web video] Nadia Hallgren.

Beginning, 1967. [Web video] Artavazd Peleshian.

Being There, 1979. [Blu-ray] Hal Ashby.

Berlin: Symphony of a Great City, 1927. [Web video] Walter

Ruttmann.

Betty Boop's Rise to Fame, 1934. [Web video] Dave Fleischer. Beyond Clueless, 2014. [DVD] Charlie Shackleton.

Bicycle Thieves, 1948. [DVD] Vittorio de Sica [screenshot only].

Bluebottles, 1928. [Web video] Ivor Montagu.

Bob Kick the Mischievous Kid, 1903. [DVD] Georges Méliès.

Bob's Burgers supercut: All of Tina's moans, 2012. [Web video]

Bryan Menegus for Slacktory.

Bodysong, 2003. [DVD] Simon Pummell.

Bop-Scotch, 1952. [Web video] Jordan Belson.

Breakfast, Lunch, and Dinner Supercut, 2018. [Web video] The Royal Ocean Film Society.

Breathless, 1959. [Blu-ray] Jean-Luc Godard.

Brian Williams Raps Snoop Dogg, 2013. [Web video] John MacDonald for The Tonight Show Starring Jimmy Fallon.

Broadway Melody of 1938, 1937. [DVD] Roy Del Ruth, montage sequence by Slavko Vorkapich.

Buffy, 2002. [Web video] Chuck Jones.

Butch Cassidy and the Sundance Kid, 1969. [DVD] George Roy Hill [screenshot only].

By Night with Torch and Spear, 1940s. [DVD] Joseph Cornell. Can Dialectics Break Bricks?, 1973. [Web video] René Viénet.

Casting JonBenet, 2017. [Web video] Kitty Green.

Cats Die Funny, Dogs Die Sad, 2016. [Web video] Jacob T.

Swinney for Fandor.

“CBS Evening News” headlines for Monday, July 30, 2018, 2018. [Web video] CBS Evening News.

CBS Evening News with Walter Cronkite, 3 Apr. 1975. [Web video] CBS Inc.

CBS Evening News with Walter Cronkite, 31 Jul. 1968. [Web video] CBS Inc.

CBS Evening News with Walter Cronkite, 25 Dec. 1978. [Web video] CBS Inc.
Chevy Trucks: Celebrating A Century of Dependability, 2017. [Web video] Chevy.

Chronicle of a Summer, 1961. [Blu-ray] Jean Rouch, Edgar Morin.

Chronovision (Time travel tribute), 2018. [Web video] Johanna Vaude for Blow Up - ARTE.

Cinema 2017: The Dance, 2017. [Web video] Jacob T. Swinney. Cinema Paradiso, 1988. [Web video] Giuseppe Tornatore.

'Cinema Paradiso' Wins Foreign Language Film: 1990 Oscars, 2013. [Web video] Oscars.

Citizen Kane, 1941. [DVD] Orson Welles.

Cleo from 5 to 7, 1962. [DVD] Agnès Varda.

CNN Concatenated, 2002. [Web video] Omer Fast.

Color Adjustment, 1991. [Web video] Marlon Riggs.

Concussion Protocol, 2018. [Web video] Josh Begley for Field of Vision.

Corporate Consolidation, 2017. [Web video] Last Week Tonight with John Oliver (HBO).

Cowboy and 'Indian' Film, 1958. [Web video] Raphael Montañez Ortíz.

Creativity Beyond Originality: György Pálfis FINAL CUT as Narrative Supercut, 2015. [Web video] Miklos Kiss [transcript only].

Credits, 1984. [VHS] Antonio Muntadas.

Daft Punk's “Get Lucky” - The Ultimate Dance Compilation, 2013. [Web video] Huffington Post UK.

Dancing in Movies - Supercut, 2014. [Web video] supercutonline. Dancing Movie montage, 2010. [Web video] Clara Darko.

dancing to the end of poverty, 2008. [Web video] Margaux Williamson.

Dara Birnbaum - Installation \& Video Artist, 2015. [Web video] School of Visual Arts [transcript only].

Dara Birnbaum: the dark matter of media light, 2009. [Web video] artvideotv.

Davey Jones' Locker, 1900. [DVD] Frederick Armitage.

David Bowie and Queen: Under Pressure music video, 1981. [Web video] David Mallet.

Days of Heaven, 1978. [Blu-ray] Terrence Malick.

Dead Men Don't Wear Plaid, 1982. [DVD] Carl Reiner.

Dead of Night, 1945. [poster] Alberto Cavalcanti, Charles Crichton, Basil Drearden, Robert Hamer.

Der verlorene Schuh, 1923. [Web video] Ludwig Berger.

Die Nibelungen, Part I. Siegfried, 1924. [DVD] Fritz Lang.

Dinosaur Movies, 1993. [Web video] Donald F. Glut [screenshots only].

Directed by John Ford, 1971. [Web video] Peter Bogdanovich.

Donald Trump Counts To The Biggest Number Ever, 2018. [Web video] VICE News.

Don't die on me - Supercut, 2014. [Web video] supercutonline.

Down the Aisle: A Wedding Supercut, 2014. [Web video] Max Tohline.

DP/30: Chuck Workman, Magician: The Astonishing Life and Work of Orson Welles, 2014. [Web video] DP/30: The Oral History Of Hollywood (David Poland).

Duck Soup, 1933. [DVD] Leo McCarey.

Dunkirk, 2017. [Web video] Christopher Nolan [screenshot only]. 
E.T.: The Extra-Terrestrial, 1982. [Web video] Steven Spielberg [screenshot only].

Echopraxia, 2005. [Web video] Fu Pok Yan.

Egypte: Panorama des Rives du Nil [Lumière cat. no. 386], 1897. [Web video] Alexandre Promio.

Elégia, 1965. [Web video] Zoltán Huszárik.

Epileptic Seizure Comparison, 1976. [Web video] Paul Sharits.

Equal+Opposite, 2008. [Web video] Rahne Alexander.

'SportsCentury' Images of Our Century montage, 1999. [Web video] ESPN Sportscenter.

Ethnic Notions, 1987. [Web video] Marlon Riggs.

Every Covid-19 Commercial is Exactly the Same, 2020. [Web video] Microsoft Sam.

Every Single Michael Bay Explosion, 2011. [Web video] Jimmy Barr.

Every Tom Cruise Run. Ever., 2016. [Web video] Burger Fiction. Every Woody Allen Stammer From Every Woody Allen Movie, 2013. [Web video] HuffPost.

Everywhere at Once, 1985. [Web video] Alan Berliner. Eyeballing, 2005. [Web video] Rosalind Nashashibi. Fahrenheit 11/9, 2018. [Web video] Michael Moore. Fahrenheit 9/11, 2004. [DVD] Michael Moore. DOI: https://doi. org/10.1353/flm.2005.0029

Fails - Hard knock Life, 2016. [Web video] BAESTI LP. Family Matters, Season 6, Episode 21 “What’s Up Doc?”, 1995. [Web video] Richard Correll.

Fantasia of Color in Early Cinema boek, 2015. [Web video] Eye Filmmuseum.

Fast Workers 2019 \#2 - Amazing Skills Level God, 2019. [Web video] United Pictures.

Fast Workers 2019 \#4 - Amazing Skills Level God, 2019. [Web video] United Pictures.

Fast Workers 2019 \#9 - Amazing Skills Level Master, 2019. [Web video] United Pictures.

Fat and Lean Wrestling Match, 1900. [DVD] Georges Méliès.

Faust, 2019. [Web video] F. W. Murnau.

Field of Dreams, 1989. [DVD] Phil Alden Robinson.

Film Before Film, 1986. [VHS] Werner Nekes.

Film in Which There Appear Edge Lettering, Sprocket Holes, Dirt Particles, Etc., 1966. [Web video] Owen Land.

Final Cut: Ladies and Gentlemen, 2012. [Web video] György Pálfi.

Fingering and Footing, 2005. [DVD] Catherine Ross.

Flying Down to Rio, 1933. [DVD] Thornton Freeland.

Footlight Parade, 1933. [DVD] Lloyd Bacon.

Forbidden Games, 1952. [DVD] Rene Clement [screenshot only].

Forrest Gump, 1994. [DVD] Robert Zemeckis.

Frank Film, 1973. [Web video] Frank \& Caroline Mouris.

Full Metal Jacket, 1987. [DVD] Stanley Kubrick.

Future Pres. Nixon on Face the Nation, 27 Oct. 1968. [Web video] CBS News.

Gabbeh, 1996. [DVD] Mohsen Makhmalbaf [screenshot only]. Gay Kiss Montage, 2006. [Web video] robe888 (Robert Eldredge). George Wallace and California: The Beginning, 1968. [Web video] Jim Guillot.

George Wallace's America and George Wallace's Americans, 1968. [Web video] director unknown.
Get Down! - Supercut, 2014. [Web video] Dan Murrell for Screen Junkies.

GET OUT OF THERE!, 2010. [Web video] hh1edits (Harry

Hanrahan).

Goals that science can't explain, 2020. [Web video] SportsHD.

God Bless the USA, 2008. [Web video] Wreck \& Salvage.

Grand Hotel, 1932. [poster] Edmund Goulding.

Gringo in Mañanaland, 1995. [Web video] DeeDee Halleck.

Grizzly Man, 2005. [DVD] Werner Herzog.

Groundhog Day, 1993. [DVD] Harold Ramis.

Groundless, ca. 2006. [Web video] Jen Proctor.

H2O, 1929. [DVD] Ralph Steiner.

Hamlet Mash Up, 2013. [Web video] Geoff Klock.

Hands, 1934. [DVD] Ralph Steiner \& Willard Van Dyke.

Hara-kiri, 1962. [Blu-ray] Masaki Kobayashi [screenshot only].

Hearts and Minds, 1974. [DVD] Peter Davis, ed. Lynzee

Klingman, Susan Martin.

Hide, 2006. [DVD] Christoph Girardet \& Matthias Müller.

His Musical Career, 1914. [Web video] Charlie Chaplin.

Hit By A Bus - The Supercut, 2013. [Web video] hh1edits (Harry Hanrahan).

Hollywood [various episodes], 1980. [Web video] Kevin Brownlow \& David Gill [screenshots].

Hollywood Justice: Prison Rape For All, 2014. [Web video] HuffPostMovieMashups.

Hollywood, Ep. 5 'Hazards of the Game', 1980. [Web video] Kevin Brownlow \& David Gill.

Holy Robin Compilation!, 2009. [Web video] tedfufu (David Irons).

Home Stories, 1990-91. [Web video] Matthias Müller. Hooper, 1978. [Web video] Hal Needham.

Horror Movie Cars Never Start When You Need Them Most, 2014. [Web video] HuffPostMovieMashups.

How Donald Trump spoke about WikiLeaks during 2016 presidential campaign, 2019. [Web video] Guardian News.

How Garfield Lost His Magic, 2018. [Web video] Quinton Reviews.

How To Curse On Network TV, 2013. [Web video] BuzzFeed Celeb. I could tell you but I'd have to kill you - a Supercut, 2010. [Web video] Oscar Bartos.

I Love Lucy, Season 6, Episode 11 “Christmas Show”, 1956. [Web video] James V. Kern.

I Love to Singa, 1936. [Web video] Tex Avery.

I, Tonya, 2017. [Web video] Craig Gillespie [screenshot only]. Ikiru, 1952. [DVD] Akira Kurosawa.

I'm Not Here to Make Friends!, 2008. [Web video] richfofo (Rich Juzwiak).

In a Nutshell, 2018. [Web video] Fabio Friedli.

In Order Not To Be Here, 2002. [DVD] Deborah Stratman.

Indy Vinyl on The Clock (and the clock), 2019. [Web video] Ian

Garwood.

Inside Llewyn Davis, 2013. [Blu-ray] Ethan and Joel Coen.

Intimate Stranger, 1991. [Web video] Alan Berliner.

Intolerance, 1916. [DVD] D. W. Griffith [screenshot only]. Iron Chef America - SECRET INGREDIENT, 2010. [Web video]

MrEh. 
It's a Mad, Mad, Mad, Mad World, 1963. [Web video] Stanley Kramer [screenshot only].

IT'S THE 90S!, 2011. [Web video] Everything is Terrible!. It's The Hard Knock Life (Fail Compilation), 2017. [Web video] Claudia Castonguay.

James, 2007. [Web video] Katharina Arndt.

Jeff Goldblum Making Noises (Supercut), 2018. [Web video] Owenergy.

Jeux des reflets et de la vitesse, 1925. [DVD] Henri Chomette. Jim Carrey Falls Down, 2015. [Web video] Shawn Kohne. Johnny Cash - Ain't No Grave [Official HD] - The Johnny Cash Project, 2010. [Web video] xChrisMilk.

Julian Palacz / Algorithmic Search for Love / Nominé Prix Cube 2013, 2013. [Web video] Le Cube.

Julianne Moore Loves to Cry..., 2010. [Web video] hh1edits (Harry Hanrahan).

Jupiter's Thunderballs, 1903. [DVD] Georges Méliès.

Keeping America Scared, 2004. [Web video] Brennan Houlihan. Keilar: Trump doesn't know WikiLeaks? Roll the tape., 2019. [Web video] CNN.

Killing Us Softly, 1979. [Web video] Margaret Lazarus \& Renner Wunderlich.

KISS KISS KISS, 1964. [Web video] Tadanori Yokoo.

Kiss Me Deadly, 1955. [DVD] Robert Aldrich.

Kiss the Girls, Make them Cry, 1979. [DVD] Dara Birnbaum. Knute Rockne: All American, 1940. [Web video] Lloyd Bacon, montage by Don Siegel.

Kramer's Entrance, 2009. [Web video] Tyler Creviston.

La classe américaine, 1993. [Web video] Michel Hazanavicius \& Dominique Mézerette.

La sortie des usines Lumière, 1895. [DVD] Auguste and Louis Lumière.

Lady Gaga saying 'there can be a hundred people in the room' for one minute straight, 2018. [Web video] The Fab Teacher. Lake Placid '80, 1980. [Web video] Nam June Paik. Landbrug i Danmark [Agriculture in Denmark], 1907. [DVD] Nordisk Films Kompagni.

Landing onto Vehicles - Supercut, 2016. [Web video] supercutonline.

Latinos Beyond Reel, 2012. [Web video] Chyng Sun \& Miguel Parker. Le commandant Marchand au Cercle Militaire à Paris [Lumière cat. no. 1032], 1899. [Web video] unknown operator. Leave No Trace, 2018. [DVD] Debra Granik [screenshot only]. Lesbian Kiss Episodes: 'Vulture's Secret History of Television' Episode 4, 2015. [Web video] New York Magazine.

"Let Her Go": The Movie Cliché Supercut, 2014. [Web video] HuffPostMovieMashups.

Let's Dance, 2010. [Web video] bnewcol.

Let's Enhance, 2009. [Web video] Duncan Robson.

Lisbôa Cronica Anedótica, 1930. [Web video] José Leitão de Barros.

Liverpool: La rade [Lumière cat. no. 702], 1897. [Web video] Alexandre Promio.

Lonesome, 1928. [Blu-ray] Paul Fejos.

Long Live the New Flesh, 2009. [Web video] Nicolas Provost. Loose Canon: Hillary Clinton, 2016. [Web video] Lindsay Ellis.
Lumière and Company, 1995. [poster] 40 directors.

Mad Men's Don Draper Says ‘What?’, ca. 2010. [Web video] dondrapersayswhat.

Make Mine Music!, 1946. [poster] Robert Cormack, Clyde Geronimi, Jack Kinney, Hamilton Luske, Joshua Meador.

Man with a Movie Camera, 1929. [DVD, Blu-ray] Dziga Vertov.

Manufacturing Consent, 1992. [Web video] Mark Achbar \& Peter Wintonick.

March of the Movies, 1933. [Web video] J. Stuart Blackton.

Marlene, 1984. [DVD] Maximilian Schell.

Marvel Studios' Captain Marvel - Official Trailer, 2018. [Web video] Marvel Entertainment.

Mary Poppins, Eliza Doolittle, and Julie Andrews’ Oscar, 2018.

[Web video] Be Kind Rewind.

MATCH CUT: The Art of Cinematic Technique, 2016. [Web video] Celia Gómez.

Mechanical Principles, 1930. [DVD] Ralph Steiner.

Media Reacts To Conan's Same-Sex Wedding News, 2011. [Web video] TeamCoco.

Melody Cruise, 1933. [DVD] Mark Sandrich.

Melody of the World, 1929. [Web video] Walter Ruttmann. Metro-Goldwyn-Mayer, 1975. [Web video] Jack Goldstein.

Metropolis, 1927. [Blu-ray] Fritz Lang.

Midnight, 2013. [Web video] Carolina Natalie [bootleg footage of an audience watching The Clock (Christian Marclay, 2010), shot at LACMA].

Montparnasse, 1929. [Web video] Eugène Deslaw.

Moskva, 1926. [Web video] Mikhail Kaufman \& Ilya Kopalin. Movie Phone Super Call, 2015. [Web video] Burger Fiction.

Movies in movies: A montage, 2013. [Web video] Clara Darko \& Brutzelpretzel for Slacktory.

My Meds, 2009. [Web video] Natalie Bookchin.

My Own Private Idaho, 1991. [DVD] Gus van Sant [screenshot only].

Myrna Loy: So Nice To Come Home, 1991 [TV] Richard Schickel. Myth in The Electric Age, 1981. [Web video] Alan Berliner.

Napoleon, 1927. [Blu-ray] Abel Gance.

Naqoyqatsi, 2002. [DVD] Godfrey Reggio.

Nashville, 1975. [poster] Robert Altman.

Nausicaa of the Valley of the Wind, 1984. [DVD] Hayao Miyazaki [screenshot only].

NBC Nightly News with Tom Brokaw, 31 Oct. 1992. [Web video] National Broadcasting Company.

Next Stop, Analysis: The Contradictory Trains of Cinema, 2018

[Web video] What's So Great About That? (Grace Lee).

Night of 1000 Eyes, 1984. [Web video] Suzanne Goldbacher \& Kim Flitcroft.

Normal Appearances, 2017. [Web video] Penny Lane.

North by Northwest, 1959. [Blu-ray] Alfred Hitchcock.

Nothing, 2017. [Web video] LJ Frezza.

October: Ten Days that Shook the World, 1927. [DVD] Sergei

Eisenstein.

Of Oz the Wizard, 2004. [Web video] Matt Bucy.

Oh Boy, 1990. [Web video] Sterling Eidolan \& the Odd Woman

Out.

Olympia, Part 2: Festival of Beauty, 1938. [DVD] Leni Riefenstahl. 
One Sixth of the World, 1926. [Web video] Dziga Vertov.

Opinion / Where's the love for WikiLeaks now, President Trump?,

2019. [Web video] Washington Post.

Our Little Sister, 2015. [DVD] Hirokazu Kore-eda.

Overlord, 1975. [DVD] Stuart Cooper.

Owen Wilson Says WOW, 2015. [Web video] Owenergy.

Paramount on Parade, 1930. [poster] 21 directors.

Paris, je t'aime, 2006. [poster] 22 directors.

People in Movies and TV Hitting Deer With Their Cars (Supercut),

2017. [Web video] Wiseguy Pictures.

People on Sunday, 1930. [Blu-ray] Robert Siodmak \& Edgar G.

Ulmer.

Perhaps, Perhaps, Perhaps, 2005. [Web video] Killa.

Place de la Comédie [no Lumière catalogue number], 1896.

[Web video] unknown operator.

Plain Talk and Common Sense, 1987. [Web video] Jon Jost.

Play, 2003. [DVD] Christoph Girardet \& Matthias Müller.

Point of Order, 1963. [Web video] Emile de Antonio.

Police appear to target journalists as they cover George Floyd

protests, 2020. [Web video] Washington Post.

Police Mortality, 2013. [Web video] Anti-Banality Union.

Precious Images, 1986 [90's re-release]. [Web video] Chuck

Workman.

Precious Images winning the Oscar $®$ for Live Action Short, 1987.

[Web video] Oscars.

Pulp Fiction, 1994. [poster] Quentin Tarantino.

Pure, 2009. [Web video] Jacob Bricca.

Rabid, 2005. [Web video] Mike Olenick.

Radetzky March Supercut (Vienna Phil New Year's compilation), 2015. [Web video] amt253 (Max Tohline).

Raiders of the Lost Ark, 1981. [DVD] Steven Spielberg

[screenshot only].

Reading Marx's 'Capital' Volume 1 with David Harvey, Part 8,

2019. [Web video] The People's Forum NYC.

Rebel Without a Cause, 1955. [DVD] Nicholas Ray [screenshot only]. REcreation, 1956-57. [Web video] Robert Breer.

Reel Bad Arabs, 2006. [Web video] Sut Jhally \& Jack Shaheen.

Reel Injun, 2010. [Web video] Catherine Bainbridge \& Neil

Diamond.

Regen [Rain], 1929. [DVD] Joris Ivens.

Relatively Romantic - 1000 Kisses, 1984. [Web video] Klaus vom Bruch.

Richard Nixon Campaign Ad 'Law and Order', 1968. [Web video] director unknown.

Richard Nixon's Acceptance Speech at the 1968 Republican

National Convention, 1968. [Web video] director unknown.

Rien que les heures, 1926. [Web video] Alberto Cavalcanti.

Ro.Go.Pa.G, 1963. [poster] Roberto Rossellini, Jean-Luc Godard,

Pier Paolo Pasolini, Ugo Gregoretti.

Rock Hudson's Home Movies, 1992. [VHS] Mark Rappaport.

Ronald McDonald insanity [M. C. Donarudo wa dansu ni

muchuna no ka? Saishu kichiku dokeshi Donarudo M], 2008.

[Web video] Mikuru.

Rose Hobart, 1936. [Web video] Joseph Cornell.

Rund um die Liebe, 1929. [censor cards in the German Federal

Archive] Oskar Kalbus.
Salaam Cinema, 1995. [Web video] Mohsen Makhmalbaf.

Samuel L. Jackson Death Reel, 2012. [Web video] Jukka-Pekka Bohm.

San Francisco, 1936. [DVD] W.S. Van Dyke.

São Paulo, a Metropolitan Symphony, 1929. [Web video]

Adalberto Kemeny \& Rudolpho Rex Lustig.

Say It with Songs, 1929. [Web video] Lloyd Bacon.

Schichlegruber Doing the Lambeth Walk, 1941. [DVD] Charles A.

Ridley.

Secrets of a Soul, 1926. [Web video] Georg Wilhelm Pabst.

Seeing Time, 2007. [Web video] Su-Chen Hung.

Seinfeld, Season 6, Episode 14 "The Highlights of a Hundred",

1995. [DVD] ed. Janet Ashikaga.

Sherlock, Jr., 1924. [DVD] Buster Keaton.

Sinclair's Soldiers in Trump's War on Media, 2018. [Web video]

Timothy Burke for Deadspin.

Sing, 2016. [Web video] Garth Jennings.

Sins of the Fathers, 1928. [DVD] Ludwig Berger, montage

sequence by Slavko Vorkapich.

Sirens, 2012. [Web video] Tyler Adams.

Sit Down and Shut Up: The Supercut, 2011. [Web video]

dondrapersayswhat.

So This is Paris, 1926. [Web video] Ernst Lubitsch.

Sofia Coppola - Gilded Cages, 2017. [Web video] Joost Broeren

$\&$ Sander Spies for Fandor.

Somewhere only we know, 2009. [Web video] Jesse McLean.

Sorkinisms - A Supercut, 2012. [Web video] Kevin T. Porter.

Sour Death Balls, 1993. [Web video] Jessica Yu.

Speedy, 1928. [Blu-ray] Ted Wilde.

Spike Lee - The Dolly Shot (Video Essay), 2012. [Web video]

Richard Cruz. DOI: https://doi.org/10.1186/1687-5281-2012-7

Spin, 1995. [DVD] Brian Springer.

Sport, Sport, Sport, 1970. [Web video] Elem Klimov.

Standard Gauge, 1984. [Web video] Morgan Fisher.

Star Wars Featurette: The Birth of the Lightsaber, 2014. [Web

video] Star Wars.

Star Wars: The Premake, 2015. [Web video] John D’Amico.

States of the Union - Bill Clinton, 1997. [Web video] Aaron Valdez.

Statues also Die, 1953. [DVD] Chris Marker.

Sumurun, 1920. [Web video] Ernst Lubitsch.

Supercut: People in movies never kiss, 2013. [Web video] Alex Moschina for Slacktory.

Supercut: Reaction Videos (turn down your headphones!), 2012.

[Web video] Slacktory.

Supercut: There's a storm coming, 2012. [Web video] Jukka-

Pekka Bohm.

Taking Off, 1971. [DVD] Milos Forman.

Teaching the Alphabet, 2007. [Web video] Volker Schreiner.

Technology/Transformation: Wonder Woman, 1978. [DVD] Dara Birnbaum.

Telephones, 1995. [Web video] Christian Marclay.

Ten Meter Tower [Hopptornet], 2016. [Web video] Axel

Danielson \& Maximilien van Aertryck.

That's Entertainment!, 1974. [DVD] Jack Haley, Jr.

The 'Best Picture' Show: The Final Image Oscar Montage, 2014.

[Web video] Monte Patterson. 
The 60 $0^{\text {th }}$ Annual Academy Awards telecast, 1988. [Web video] Marty Pasetta, montage by Chuck Workman.

The $62^{\text {nd }}$ Annual Academy Awards telecast, 1990. [Web video] Jeff Margolis, montage by Chuck Workman.

The $64^{\text {th }}$ Annual Academy Awards telecast, 1992. [Web video] Jeff Margolis, montage by Chuck Workman.

The $70^{\text {th }}$ Annual Academy Awards telecast, 1998. [Web video] Louis J. Horvitz, montage by Chuck Workman.

The $82^{\text {nd }}$ Annual Academy Awards telecast, 2010. [Web video] Hamish Hamilton, montage by Chuck Workman.

The Adorkable Misogyny of The Big Bang Theory, 2017. [Web video] Pop Culture Detective (Jonathan McIntosh).

The Adventures of Mark Twain, 1944. [DVD] Irving Rapper, montage sequences by Don Siegel.

The Annie Gasp: A ‘Community’ Supercut, 2012. [Web video] Zach Prewitt.

The Artwork in the Age of its Mechanical Reproducibility by Walter Benjamin as told to Keith Sanborn, 1996. [Web video] Keith Sanborn.

The Atomic Café, 1982. [DVD] Jayne Loader, Kevin Rafferty, \& Pierce Rafferty.

The Automatic Moving Company, 1912. [Web video] Romeo Bossetti.

The Banner of Youth, 1957. [DVD] Walerian Borowczyk \& Jan Lenica.

The Beaches of Agnes, 2008. [DVD] Agnès Varda.

The Big Lebowski, 1999. [DVD] Ethan and Joel Coen [screenshot only].

The Big Lebowski Fucking Short Version, 2006. [Web video] FUTURE.

The Big Parade of Comedy, 1964. [TV] Robert Youngson (clip from The Cameraman (Edward Sedgwick \& Buster Keaton, 1928)).

The Big Short, 2015. [Blu-ray] Adam McKay, ed. Hank Corwin.

The Celluloid Closet, 1995. [Web video] Rob Epstein \& Jeffrey Friedman.

The Cook in Trouble, 1904. [DVD] Georges Méliès.

The Cool Girl Trope, Explained, 2019. [Web video] The Take (Susannah McCullough \& Debra Minoff).

The Corporation, 2003. [DVD\} Mark Achbar \& Jennifer Abbott.

The Cranes are Flying, 1957. [DVD] Mikhail Kalatozov [screenshot only].

The Crowd, 1928. [TV] King Vidor.

The Cruise of the Jasper B., 1926. [DVD] James W. Horne.

The Cry of the Children, 1912. [DVD] George O. Nichols.

The Cutting Edge - The Magic of Editing, 2004. [Web video] Wendy Apple.

The Daily Show with Jon Stewart, 1 Aug. 2011. [Web video] Chuck O'Neil.

The Daily Show with Jon Stewart, 11 Aug. 2011. [Web video] Chuck O'Neil.

The Daily Show with Jon Stewart, 18 Aug. 2011. [Web video] Chuck O'Neil.

The Daily Show with Jon Stewart, 27 Jan. 2011. [Web video] Chuck O'Neil.

The Daily Show with Jon Stewart, 28 Apr. 2003. [Web video] Chuck O'Neil.
The Daily Show with Jon Stewart, 28 Jun. 2011. [Web video] Chuck O'Neil.

The Dance of Life, 1929. [Web video] John Cromwell \& A. Edward Sutherland.

The Dick Van Dyke Show, Season 5, Episode 32 "The Last Chapter", 1966. [Web video] Jerry Paris \& John Rich.

The Dizzying Effect of the Snorricam, 2018. [Web video] Bill Rwehera for Fandor.

The Docks of New York, 1928. [DVD] Josef von Sternberg.

The Fabulous Baker Boys, 1989. [Web video] Steve Kloves.

The Fall of the Romanov Dynasty, 1927. [Web video] Esfir Shub.

The Family Album, 1988. [Web video] Alan Berliner.

The Famous Box Trick, 1898. [DVD] Georges Méliès.

"The Greatest" - a multifandom vid of characters of color in recent SF/F/horror, by bironic, 2018. [Web video] closetfan.

The Green Fog, 2017. [Web video] Guy Maddin, Evan Johnson \& Galen Johnson.

The Hollywood Revue of 1929, 1929. [poster] Charles Reisner.

The Human Face is a Monument, 1965. [Web video] Stan VanDerBeek.

The Infernal Cauldron, 1903. [DVD] Georges Méliès.

The Kiss, 1985. [Web video] Raphael Montañez Ortíz.

The Lost World, 1925. [Web video] Harry O. Hoyt.

The Love Goddesses, 1965. [DVD] Saul J. Turell.

The Lumière Brothers' First Films (1895-1897). 1999. [DVD] Kino. (Narrated by Bertrand Tavernier).

The Man Who Shot Liberty Valance, 1962. [DVD] John Ford [screenshot only].

The Man with the Rubber Head, 1901. [DVD] Georges Méliès.

The Man You Loved To Hate, 1979. [VHS] Patrick Montgomery.

The Many Deaths of Steve Buscemi, 2011. [Web video] dondrapersayswhat.

The March of the Movies, 1927. [Web video] Terry Ramsaye \& Otto Nelson.

The March of Time, Vol. 5 No. 12 “The Movies March On!”, 1939. [Web video] The March of Time.

The Mashin' of the Christ, 2004. [Web video] Negativland.

The Media Project, 1991. [DVD] Peter Watkins.

The Mermaid, 1904. [DVD] Georges Méliès.

The Movie Orgy, 1968. [Web video] Joe Dante.

The Movie Phone Montage - Movie Mashup, 2015. [Web video] Sebastien Kerroumi.

The Muppet Movie, 1979. [DVD] James Frawley [screenshot only].

The nightmare videos of childrens' YouTube - and what's wrong with the internet today, 2018. [Web video] James Bridle \& TED.

The Old and the New, 1929. [Web video] Grigoriy Aleksandrov \& Sergei Eisenstein.

The Republic Pictures Story, 1991. [VHS] Len Morris.

The Right Stuff, 1983. [Blu-ray] Philip Kaufman.

The Road Movie [Doroga], 2016. [Web video] Dmitrii Kalashnikov.

The Robe, 1953. [DVD] Henry Koster [screenshot only].

The Secret Policeman's Ball, 1979. [poster] Roger Graef.

The Slanted Screen, 2006. [Web video] Jeff Adachi.

The Slow Clap, 2007. [Web video] YuppiePunk. 
The Spencer Tracy Legacy: A Tribute by Katharine Hepburn, 1986. [TV] David Heeley.

The Sweetest Sound, 2001. [Web video] Alan Berliner. The Ten Commandments, 1923. [Web video] Cecil B. DeMille.

The View: A 'Back-to-the-Camera Shot' Montage, 2013. [Web video] Zach Prewitt.

The Wilhelm Scream, 2011. [Web video] Cinexcellence.

The Wire: Tautology Supercut, 2015. [Web video] Propolandante.

The Wizard of Oz, 1939. [DVD] Victor Fleming, et al [screenshot only].

Things - Zoetrope, 2008. [Web video] bre pettis.

Thumbs up in the movies, 2017. [Web video] Onikorp.

To the Happy Few, 2003. [DVD] Thomas Draschan \& Stella Friedrichs.

Tom and Jerry, Ep. 66: Smitten Kitten, 1952. [Web video] William Hanna \& Joseph Barbera.

Tom Tom, The Piper's Son, 1969. [Web video] Ken Jacobs.

Touki Bouki, 1973. [Blu-ray]. Djibril Diop Mambety [screenshot only].

Trump Dodges Questions on Mueller, Tax Returns, WikiLeaks - A Closer Look, 2019. [Web video] Late Night with Seth Meyers.

Trump offers no opinion on WikiLeaks or Assange, 2019. [Web video] Fox News.

Trump's WikiLeaks Amnesia, 2019. [Web video] Mother Jones. Tucker: Our leaders dither as our cities burn (GRAPHIC VIDEO), 2020. [Web video] Fox News.

Turksib, 1929. [Web video] Victor A. Turin.

Twelve Hundred Ghosts - A Christmas Carol in Supercut, 2016. [Web video] Heath Waterman.

Unsubscribe Series \#1 - Special Offer Inside, 2010. [DVD] Jodie Mack. Untitled (Potential Terrorist), 2002. [Web video] Kerry Tribe.

Uomini, anni, vita [People, Years, Life], 1990. [Web video] Yervant Gianikian \& Angela Ricci Lucchi.

Very Nice, Very Nice, 1961. [Web video] Arthur Lipsett.

Visual Disturbances, 2018. [Web video] Eric Faden.

Viva Villa, 1934. [DVD]Jack Conway, Howard Hawks, \& William Wellman, montage sequence by Slavko Vorkapich.

Wail, 1960. [DVD] Jeff Keen.

Ways of Seeing, Ep. 2, Women in Art, 1972. [Web video] John Berger \& Michael Dibb.

Ways of Seeing, Ep. 3, Collectors and Collecting, 1972. [Web video] (John Berger \& Michael Dibb.

Ways of Seeing, Ep. 4, Commercial Art, 1972. [Web video] John Berger \& Michael Dibb.

We Will Rock You, ca. 1992. [Web video] Emergency Broadcast Network.

Weekend Update - Julian Assange Arrested, 2019. [Web video] Saturday Night Live.

Wes Anderson // Centered, 2014. [Web video] kogonada

Westinghouse Works, Panorama View Street Car Motor Room, 1904. [DVD] Billy Bitzer.

We've Got Company! from The Guy Bauer Half Hour, 2010. [Web video] Guy Bauer.

What do you do with a drunken Vulcan?, 2012 re-creation of 1975 slide show. [Web video] Kandy Fong.
What Trump has said about Wikileaks - BBC News, 2019. [Web video] BBC News.

What's Up, Tiger Lily?, 1967. [DVD] Woody Allen.

Whitsuntide Fair at Preston, 1906. [DVD] Mitchell and Kenyon.

Wide Awake, 2006. [Web video] Alan Berliner.

Won't You Be My Neighbor, 2018. [Web video] Morgan Neville.

Wonder Woman Spins! - First Season, 2007. [Web video] olsolino.

Wouldn't It Be Nice?, 2002. [Web video] Laura Shapiro.

You Bet Your Life outtakes reel, ca. 1950-52, [Web video] compiler unknown.

You have beautiful stairs, you know, 1986. [DVD] Agnès Varda.

“You Just Don't Get It, Do You?": A Montage of Cinema's Worst Writing Cliche, 2012. [Web video] Jeff Smith.

You're watching Fox News. You just don't know it., 2019. [Web video] Carlos Maza for Vox.

Youtube Poop - Aladdin commits suicide, 2010. [Web video] AlvinYTP.

Zorns Lemma, 1970. [Blu-ray] Hollis Frampton.

\section{OTHER ARTWORKS}

A-E-I-O-U and Sometimes Y, 2009. [Plastic rhinestones, acrylic, and enamel on panel] Mickalene Thomas.

algorithmic search for love, 2010. [Keyboard, computer, screens, software] Julian Palacz.

All, 2010. [Installation of the artist's complete body of work at the Guggenheim Museum, NYC] Maurizio Cattelan.

Amazing Grace, 1993. [Baby strollers, fire hose, and audio] Nari Ward.

Art Bin, 2010. [Installation of discarded artworks] Michael Landy.

Atlas, 1962-ongoing. [Found photographs on over 800 sheets] Gerhard Richter.

Black Drawings, 1991-92. [Mixed media on paper in 112 parts] Marlene Dumas.

Boulevard du Temple, 1838. [Daguerreotype] Louis Daguerre.

Car Atlas - Rainbow, 2010. [Installation of 2500 toy cars] David T. Waller.

Chorus II, 1988. [29 framed photographs] Christian Marclay.

Cloister of Saint-Trophime, Arles, 1851. [Partly hand-painted paper print composited from 10 separate negatives] Edouard Baldus. (with diagram of compositing lines from Marien, M W 2006 Photography: A Cultural History, 2nd Ed. Pearson Prentice Hall, p. 58.)

Coal Bunkers, Frontal Views, 1986-93. [Nine silver prints] Bernd $\&$ Hilla Becher.

Cooling Towers, Steel-Corrugated Concrete: Cologne, Ruhr District, 1959-72. [Nine silver prints] Bernd \& Hilla Becher.

Crying Divas from the Screenplays of an Embroiderer II, 1999. [Laser prints on canvas with metallic embroidery] Francesco Vezzoli.

Cut with the Kitchen Knife through the Beer-Belly of the Weimar Republic, 1919. [Collage of pasted papers] Hannah Höch.

Every Mickey, 2017. [SLS Nylon, Acrylic Paint, STL File] Matthew Plummer Fernandez.

Every Shot, Every Episode, 2001. [277 video compact discs and video playback installation] Jennifer and Kevin McCoy. 
Every... Bernd and Hilla Becher Gable Sided House, 2004. [Digital chromogenic print] Idris Khan.

Framework Houses, Industrial District of Siegen, 1959-1973, 1993. [12 duotone offset prints] Bernd \& Hilla Becher.

Hurray, the Butter is All Gone!, 1935. [Photocollage] John Heartfield.

ID400 (\#201-300), 1998-2001. [Gelatin silver prints] Tomoko Sawada.

Infinity of Typewriters and Infinity of Monkeys and Infinity of Time $=$ Hamlet, 1962. [Mixed media with typewriters] Arman.

John 3:16, 2000. [Single-channel video installation] Paul Pfeiffer.

Jorge Luis Borges (Book of Sand), 1995. [Lithograph] Joseph Kosuth.

Mesopotamian Dramaturgies, 2009. [Video installation] Kutluğ Ataman.

Minster, 1987-90. [Car parts] Tony Cragg.

Museums series, 1999. [Array of found objects mounted in artist's box] Barton Beneš.

My Things: Book Keeping, 2006. [Digital print] Hong Hao.

No Man's Land [selected images], 2011-2013. [Digital images] Mishka Henner.

Portrait of Robert Bonner, ca. 1860s. [Albumen silver print] William H. Mumler.

Rough Seas, 2012-14. [20 archival dry prints] Susan Hiller. Selos, 1999. [Collaged stamps on paper] Nelson Leirner.

Signs without Signification, 2003-2007. [24 archival pigment prints] Jeff Brouws.

Sixteen Jackies, 1964 [Acrylic and silkscreen ink on canvas] Andy Warhol.

Solo Scenes, 1998. [131 VHS tapes on 128 monitors with VCRs, shelving] Dieter Roth.

Some Homosexual History, Profiles, Assumptions 'Nach Magnus Hirsfeld,' 2008. [Mixed media installation] Henrik Olesen.

Synecdoche, 1991-present. [Oil and wax on wood] Byron Kim.

The Brain, 2000-2014. [Mixed-media installation] Douglas Coupland.

The Construction of Fear [details], 2008. [Coupler prints] Antoni Muntadas.

The Horse in Motion, 1879. [Cabinet card] Eadweard Muybridge.

The hot winds that blow from the West, 2011. [Construction of 131 radiators] Bharti Kher.

The Nazis, 1998. [164 chromogenic photographs] Piotr Uklański.

The SHAPES Project, 2006. [7,056 unique digital prints, $4.25 \mathrm{x}$ 5.5 in each] Allan McCollum.

The Sleepers, 1979. [176 photographs and 23 texts] Sophie Calle.

Tide Chandelier, 2005. [Found objects washed up on seashores] Stuart Haygarth.

Twentysix Gasoline Stations [selected images], 1963. [Artist's book] Ed Ruscha.

Twentysix Gasoline Stations, 2009. [26 C-prints on aluminum] Jan Freuchen.

Two-Headed Man, c. 1855. [Daguerreotype] Unidentified American artist.
Untitled (Blue Objects), 2009. [Mixed media installation] Peter Coffin.

Untitled (Tokyo), 1988-2005. [Chromogenic prints] Naoya Hatakeyama.

Visible World, 1987-2001. [3000 color photograph transparencies on 15 light-box tables] Fischli \& Weiss.

Voluntary Tortures, 1972 [86 gelatin silver prints] Annette Messager.

Waste Not, 2006. [Installation of 10,000 objects owned by the artist's mother] Song Dong.

Water Towers, 1965-97. [15 Gelatin silver prints] Bernd \& Hilla Becher.

Water Towers, 1972. [Nine silver prints] Bernd \& Hilla Becher. Water Towers, 1988. [21 silver prints] Bernd \& Hilla Becher. Water Towers, 1988. [Nine gelatin silver prints] Bernd \& Hilla Becher.

Witness, 2007. [Multimedia video installation] Frances Bagley. Workers Leaving the Factory in 11 Decades, 2006. [12-channel video installation] Harun Farocki.

You see I am here after all, 2008. [3,883 vintage postcards] Zoe Leonard.

\section{OTHER IMAGE CREDITS}

'abc world news tonight 1975,' 2020, YouTube, search result, viewed 23 June 2020, https://www.youtube.com/ results?search_query=abc+world+news+tonight+1975.

Amazon Mechanical Turk 2020, Homepage, viewed 14 July 2020, https://www.mturk.com/.

Ansari, A \& Mohammed, M 2015 Content based video retrieval systems-methods, techniques, trends and challenges. International Journal of Computer Applications 112.7.

Ansyari, I, n.d. World National Flags of Countries, available at https://www.dreamstime.com/collection-set-illustratorvector-flags-world-their-names-isolated-white-backgroundworld-national-flags-image139636937 [Accessed 7 December 2020].

Asha, D, Madhavee Latha, Y, \& Reddy, V S K 2018 Content based video retrieval system using multiple features. International Journal of Pure and Applied Mathematics 118(14), pp. 287-294.

'Automatic number-plate recognition,' 2020, Wikipedia, wiki article, viewed 12 July 2020, https://en.wikipedia.org/wiki/ Automatic_number-plate_recognition.

Avgerinakis, K, Moumtzidou, A, Galanopoulos, D, Orfanidis, G, Andreadis, S, Markatopoulou, F, Batziou, E, Ioannidis, K, Vrochidis, S, Mezaris, V, \& Kompatsiaris, I 2018 ITICERTH participation in TRECVID 2018. National Institute of Standards and Technology. Available at: https://www-nlpir. nist.gov/projects/tvpubs/tv18.papers/iti_certh.pdf [Accessed 23 December 2020].

Awad, G, Fiscus, J, Joy, D, Michel, M, Smeaton, A, et al 2017 TRECVID 2016: Evaluating video search, video event detection, localization, and hyperlinking, paper presented at TRECVID, November 2016, Available at: https://hal. archives-ouvertes.fr/hal-01854776/document [Accessed 24 December 2020]. 
Bansal, R, \& Chakraborty, S 2019 Visual content based video retrieval on natural language queries, paper presented at the 34th ACM/SIGAPP Symposium on Applied Computing, April 2019. DOI: https://doi.org/10.1145/3297280.3297303

Barthel, K U, Hezel, N, \& Mackowiak, R 2015 Graph-based browsing for large video collections. Paper presented at International Conference on Multimedia Modeling. Springer, Cham, 2015, available at: https://link.springer.com/ chapter/10.1007/978-3-319-14442-9_21 [Accessed 24 December 2020]. DOI: https://doi.org/10.1007/978-3-31914442-9_21

BBC 2020 Class to remove 'sexist' dictionary definitions for 'woman'. BBC 4 March 2020, Available at https://www.bbc. com/news/uk-england-oxfordshire-51738824 [Accessed 7 December 2020].

Bonte, A 2002 Happy End für eine Filmbibliothek? Die Filmbibliothek Kalbus in der Universitätsbibliothek Heidelberg. In: Theke. Informationsblatt der Mitarbeiterinnen und Mitarbeiter im Bibliothekssystem, (2002), pp. 27-32.

Bourgeois, L 1998 Sunday Afternoons: A Conversation and a Remark on Beauty. In: Beckley, B \& Shapiro, D (eds.) Uncontrollable Beauty: Toward a New Aesthetics. New York: Allworth Press.

'Cell site,' 2020, Wikipedia, wiki article, viewed 12 July 2020, https://en.wikipedia.org/wiki/Cell_site.

Chen, J, Huang, P, Liu, J, Liang, J, Hu, T, Ke, W, Barrios, W, Vaibhav, V, Chang, X, Huang, D, \& Hauptmann, A 2018 Informedia @ TRECVID 2018: Ad-hoc Video Search, Video to Text Description, Activities in Extended video. National Institute of Standards and Technology. Available at: https:// www-nlpir.nist.gov/projects/tvpubs/tv18.papers/inf.pdf [Accessed 23 December 2020].

'Claude Closky, b. 1963,' 2020, ubu.com, director page, viewed on 23 June 2020, https://ubu.com/film/closky_mouths.html.

'Closed-circuit television,' 2020, Wikipedia, wiki article, viewed 12 July 2020, https://en.wikipedia.org/wiki/Closed-circuit_ television.

Cushing, E 2013 Amazon Mechanical Turk: The Digital Sweatshop. Utne Reader, Jan/Feb 2013, Available at: https://www.utne.com/science-and-technology/amazonmechanical-turk-zm0z13jfzlin [Accessed 14 July 2020].

Databank Data Centers, Bluffdale, UT, to National Archives Museum in Washington, D.C., 2020, Google Maps, route overview, 8 July 2020, https://www.google.com/maps/ dir/DataBank+Data+Centers,+14944+Pony+Express+Rd, +Bluffdale,+UT+84065/National+Archives+Museum ,+Constitution+Avenue+Northwest,+Washington,+ DC/@40.5691884,-111.980213,11z/data=!4m14!4m1 3!1m5!1m1!1s0x8752811fcae8732d:0x89e857ad71 f15c70!2m2!1d-111.904533!2d40.480032!1m5!1m1 !1s0x89b7b79adcbc14d1:0x17b51a59f16166f1!2m2!1d-77.022939!2d38.892559!3e0.

de Oliveira Barra, G, Lux, M, \& and Giro-i-Nieto, X. 2016 Large scale content-based video retrieval with LIVRE. Paper presented at the 14th International Workshop on Content-
Based Multimedia Indexing (CBMI). IEEE, June 2016. DOI: https://doi.org/10.1109/CBMI.2016.7500266

Dürer, A 1538 Underweysung der Messung, 2nd ed., Nuremberg. Available at https://www.metmuseum.org/art/collection/ search/336657 [Accessed 24 December 2020].

'Dylan Marron,' 2020, YouTube, channel page, viewed 9 June 2020, https://www.youtube.com/user/dylanmarron/videos.

'every f word,' 2019, YouTube, search result, viewed 6 January 2019, https://www.youtube.com/results?search_ query=every $+f+$ word.

'E-ZPass,' 2020, Wikipedia, wiki article, viewed 12 July 2020, https://en.wikipedia.org/wiki/E-ZPass.

E-ZPass New York Service Center 2020 E-ZPass Info: Plans, viewed 12 July 2020, https://www.e-zpassny.com/en/about/ plans.shtml.

E-ZPass New York Service Center 2020 E-ZPass Quick Guide, viewed 12 July 2020, https://www.e-zpassny.com/en/ about/i_guide.pdf.

Francis, D, Huet, B, \& Merialdo, B 2018 EURECOM participation in TrecVid VTT 2018. National Institute of Standards and Technology. Available at: https://www-nlpir.nist.gov/ projects/tvpubs/tv18.papers/eurecom.pdf [Accessed 23 December 2020].

Gebauer, L; Kringelbach, M L; and Vuust, P 2012 Ever-Changing Cycles of Musical Pleasure: The Role of Dopamine and Anticipation. Psychomusicology: Music, Mind, and Brain, 22(2): 152-167. DOI: https://doi.org/10.1037/a0031126

'Global Positioning System,' 2020, Wikipedia, wiki article, viewed 12 July 2020, https://en.wikipedia.org/wiki/Global_ Positioning_System.

Google 2020 Homepage, various search queries, viewed 14 July 2020, https://www.google.com/.

Haberman, M 2018, Twitter update, 1 April, viewed 3 April 2018, https://twitter.com/maggienyt/ status/980600512256110598?lang=en.

'Henny Porten - Leben und Laufbahn einer Filmkünstlerin,' 2020, IMDb, title entry, viewed 23 June 2020, https://www. imdb.com/title/tt1348972.

Hong, S, Im, W, \& Yang, H 2018 CBVMR: content-based videomusic retrieval using soft intra-modal structure constraint. Paper presented at the 2018 ACM on International Conference on Multimedia Retrieval, June 2018. DOI: https:// doi.org/10.1145/3206025.3206046

Iqbal, S, Qureshi, A, \& Lodhi, A 2018 Content Based Video Retrieval Using Convolutional Neural Network. In: Arai, K, Kapoor, S, \& Bhatia, R (eds) Intelligent Systems and Applications. IntelliSys 2018. Advances in Intelligent Systems and Computing, vol 868. Springer, Cham. DOI: https://doi. org/10.1007/978-3-030-01054-6_12

'jay leyda films beget films,' 2020, Missouri S\&T's Curtis Laws Wilson Library, online search result, viewed 23 June 2020, http://merlin.lib.umsystem. edu/search $55 /$ ?searchtype $=Y \&$ searchscope $=5 \&$ searcharg=jay+leyda+films+beget+films.

Jiang, L, Yu, S, Meng, D, Yang, Y, Mitamura, T, \& Hauptmann, A 2015 Fast and accurate content-based semantic search 
in $100 \mathrm{~m}$ internet videos, paper presented at the $23 \mathrm{rd} \mathrm{ACM}$ international conference on Multimedia, October 2015. DOI: https://doi.org/10.1145/2733373.2806237

Jin, Y, Kwak, J, Lee, Y, Yun, J, \& Ko, H 2018 KU-ISPL TRECVID 2018 VTT Model. National Institute of Standards and Technology. Available at: https://www-nlpir.nist.gov/ projects/tvpubs/tv18.slides/ku_ispl.vtt.slides.pdf [Accessed 23 December 2020].

Keaton, B 1924 production still from Sherlock, Jr. In: Academy of Motion Picture Arts and Sciences 2012, Inside the Booth - A Journey Through Projection, available at: https://www. oscars.org/videos-photos/inside-booth-journey-throughprojection [Accessed on 22 December 2020].

Kircher, A 1671 Illustration of Magic Lantern. In: Ars Magna Lucis et Umbrae, p. 768, available at: https://en.wikipedia. org/wiki/Magic_lantern\#/media/File:Optic_Projection_ fig_404.jpg [Accessed 22 December 2020].

'Kiss the Girls, Make Them Cry (Dara Birnbaum, 1979),' 2020, Video Data Bank, video page, viewed 23 June 2020, https:// vdb.org/titles/kiss-girls-make-them-cry.

Kurzweil, R 2013 How to create a mind: The secret of human thought revealed. New York: Penguin.

Lavater, J K c. 1780 Essays on Physiognomy, engraving of Lavater's Silhouette Machine, Available at https:// en.wikipedia.org/wiki/Johann_Kaspar_Lavater\#/media/ File:Lavater's_Apparatus_for_Taking_Silhouettes.-(From_an_ancient_engraving_of_1783).jpg [Accessed 24 December 2020].

Levi-Strauss, C 1966 The Savage Mind. Chicago: University of Chicago Press.

'Marilyn,' 2020, IMDb, title entry, viewed 23 June 2020, https:// www.imdb.com/title/tt0057290/.

'Mass surveillance,' 2020, Wikipedia, wiki article, viewed 12 July 2020, https://en.wikipedia.org/wiki/Mass_surveillance

Mattson, M P 2014 Superior pattern processing is the essence of the evolved human brain. Frontiers in Neuroscience, 2014(8): 265. DOI: https://doi.org/10.3389/ fnins.2014.00265

Mehrotra, D 2020 Horror Stories from Inside Amazon's Mechanical Turk. Gizmodo, 28 Jan. 2020, Available at: https://gizmodo.com/horror-stories-from-inside-amazonsmechanical-turk-1840878041 [Accessed 14 July 2020].

Merriam-Webster's Collegiate Thesaurus, $2^{\text {nd }}$ Ed. 2018 Springfield, Massachusetts: Merriam-Webster, Inc.

'Movie Lists,' 2019, YouTube, playlist for the channel Cinefix, viewed 6 January 2019, https://www.youtube.com/ playlist?list=PL1AXWu-gGX6IcOGt0yaA9Cd_hkt_ TwQIC.

'Movie Phone Super Call,' 2020, Vimeo, comments section, viewed 15 June 2020, https://vimeo.com/139265123.

Mühling, M, Meister, M, Korfhage, N, Wehling, J, Hörth, A, Ewerth, R \& Freisleben, B 2019 Content-based video retrieval in historical collections of the German Broadcasting Archive. International Journal on Digital Libraries 20(2), pp. 167-183. DOI: https://doi.org/10.1007/ s00799-018-0236-z
Muybridge, E 1893 A Couple Waltzing [Phenakistoscope disc], animated, Available at: https://en.wikipedia.org/wiki/ File:Phenakistoscope_3g07690b.gif [Accessed 22 December 2020].

National Film Archive Catalogue, Part II: Silent Non-Fiction Films, 1895-1934 1960 London: The British Film Institute, Available at https://archive.org/details/ nationalfilmarch00nati [Accessed 5 December 2020].

Nelson's Foundational Bible Concordance: King James Version 2004 Nashville: Thomas Nelson.

Newman, A 2019 I Found Work on an Amazon Website. I Made 97 Cents an Hour. The New York Times, 15 Nov. 2019, Available at: https://www.nytimes.com/ interactive/2019/11/15/nyregion/amazon-mechanical-turk. html [Accessed 14 July 2020].

Niver, K 1967 'Davey Jones' Locker,' Bergsten, B (ed.), Motion Pictures from the Library of Congress Paper Print Collection, 1894-1912, Berkeley: University of California Press, p. 325. DOI: https://doi.org/10.1525/9780520334106

'Oulipo,' 2020, Wikipedia, wiki article, viewed 28 June 2020, https://en.wikipedia.org/wiki/Oulipo.

Pouyanfar, S, Tian, H, Reyes, MP, Wang, T, Cen, H, Li, Y, \& Chen, S 2018 Florida International University - University of Miami TRECVID 2018. National Institute of Standards and Technology. Available at: https://www-nlpir.nist.gov/ projects/tvpubs/tv18.papers/fiu-um.pdf [Accessed 23 December 2020].

Poyet, L 1882, Émile Reynaud's Projecting Praxinoscope. In: La Nature, revue des sciences, no. 492 (1882), p. 357, available at: https://en.wikipedia.org/wiki/File:Lanature1882_ praxinoscope_projection_reynaud.png [Accessed 22 December 2020].

'Precious Images,' 2020, Vimeo, search result, viewed 23 June 2020, https://vimeo.com/search?q=precious\%20images

'Queen Sono - User Ratings,' 2020, IMDb, user ratings page, viewed 8 July 2020, https://www.imdb.com/title/tt9426290/ ratings.

Quénot, G \& Awad, G 2018 TRECVID 2018 Ad-hoc Video Search Task. National Institute of Standards and Technology. Available at: https://www-nlpir.nist.gov/projects/tvpubs/tv18. slides/tv18.avs.slides.pdf [Accessed 23 December 2020].

Raphael Montañez Ortíz, Newsreel, 1958,' 2020, Smithsonian American Art Museum object record, viewed 24 June 2020, https://americanart.si.edu/artwork/newsreel-80356.

Rather, D 2018, Facebook update, 2 April, viewed 2 April 2018, https://www.facebook.com/24085780715/posts/ lets-be-clear-news-anchors-looking-into-camera-andreading-a-script-handed-down-/10160239588295716/.

Rodler, H 1531 A Fine, Useful Booklet on the Art of Measuring, 1531, Available at https://archive.org/stream/ eynschnntzlichbc00rodl\#page/n95/mode/2up [Accessed 24 December 2020].

Rossetto, L, Giangreco, I, Schuldt, H, Dupont, S, Seddati, O, Sezgin, M, \& Sahillioğlu, Y 2015 IMOTION_a content-based video retrieval engine. Paper presented at International Conference on Multimedia Modeling, Springer, Cham, 
January 2015, Available at: http://edoc.unibas.ch/dok/ A6329133 [Accessed 24 December 2020]. DOI: https://doi. org/10.1007/978-3-319-14442-9_24

'Satellite navigation device,' 2020, Wikipedia, wiki article, viewed 12 July 2020, https://en.wikipedia.org/wiki/Satellite_ navigation_device.

Semuels, A 2018 The Internet is Enabling a New Kind of PoorlyPaid Hell. The Atlantic, 23 Jan. 2018, Available at: https:// www.theatlantic.com/business/archive/2018/01/amazonmechanical-turk/551192/ [Accessed 14 July 2020].

'Slavko Vorkapich,' 2020, IMDb, name entry, viewed 14 July 2020, https://www.imdb.com/name/nm0903465/.

Soboroff, I, Butt, A, Curtis, K, Ellis, A, Dimmick, D, Fiscus, J, Lee, Y, Godil, A, Joy, D, \& Delgado, A 2018 TREC Video Retrieval Evaluation (TRECVID) Introduction. National Institute of Standards and Technology. Available at: https://www-nlpir. nist.gov/projects/tvpubs/tv.pubs.18.org.html [Accessed 23 December 2020].

Standage, T 2018, Twitter update, 10 September, viewed 13 December 2020, https://twitter.com/tomstandage/ status/1039230780462047232 (1784 illustration of Wolfgang von Kempelen's Mechanical Turk).

'Supercut.org,' 2020, Internet Archive's Wayback Machine, search result, viewed 15 June 2020, https://web.archive. org/web/*/supercut.org.

'The African Queen - User Ratings,' 2020, IMDb, user ratings page, viewed 8 July 2020, https://www.imdb.com/title/ tt0043265/ratings.

The Bible 1885 Revised King James Version, Oxford: Oxford University Press.

The March of the Movies 1927 National Board of Review Magazine II(3) March 1927, p. 3.

Thomanek, R, Roschke, C, Platte, B, Rolletschke, T, Heinzig, M, Vodel, M, Zimmer, F, \& Ritter, M 2018 University of Applied Sciences Mittweida and Chemnitz University of Technology at TRECVID 2018. National Institute of Standards and Technology. Available at: https://www-nlpir.nist.gov/ projects/tvpubs/tv18.papers/hsmw_tuc.pdf [Accessed 23 December 2020].

'Toll road,' 2020, Wikipedia, wiki article, viewed 12 July 2020, https://en.wikipedia.org/wiki/Toll_road.

'Traffic enforcement camera,' 2020, Wikipedia, wiki article, viewed 12 July 2020, https://en.wikipedia.org/wiki/Traffic enforcement_camera.
'Transponder,' 2020, Wikipedia, wiki article, viewed 12 July 2020, https://en.wikipedia.org/wiki/Transponder.

Tribe, K 2002 Untitled (Potential Terrorist), Detail: casting notice, viewed 5 December 2020, http://www.kerrytribe.com/ project/untitled-potential-terrorist/.

'truth, knowledge, data, information,' 2020, Google Books Ngram Viewer, search result, viewed on 8 July 2020, Available at https://books.google.com/ ngrams/graph?content=truth\%2Cknowledge\%2Cdata $\% 2$ Cinformation\&year_start=1800\&year_ end $=2000 \&$ corpus $=26 \&$ smoothing $=3$.

'UbuWeb: Film \& Video,' 2020, ubu.com, subject page, viewed on 23 June 2020, https://ubu.com/film/index.html.

Varley, C 1830 Artist Sketching with a Wollaston Camera Lucida, In: Dollond, G The Camera Lucida, London. Available at http://vision.mpiwg-berlin. mpg.de/vision_coll/imageCollection/vision_images/ zogiVisionDetails?filename=Dollond_title_cl_drawer [Accessed 24 December 2020].

'Vehicle registration plate,' 2020, Wikipedia, wiki article, viewed 12 July 2020, https://en.wikipedia.org/wiki/Vehicle_ registration_plate.

'WatchMojo.com,' 2019, YouTube, channel page, viewed 6 January 2019, https://www.youtube.com/user/WatchMojo/ videos.

Workers of the Writers' Program of the Work Projects Administration in the City of New York 1941 The Film Index: A Bibliography, Vol. 1: The Film as Art. New York: H. W. Wilson Co.

'World of Signs,' 2020, YouTube, playlist for the channel World of Signs, viewed 30 May 2020, https://www.youtube.com/ playlist?list=PLDeOH4NU6Cm4FRkx_OrHAw18RuVwSPj-h [Accessed 30 May 2020].

Yu, S, Jiang, L, Xu, Z, Yang, Y, Hauptmann, A 2015 Contentbased video search over 1 million videos with 1 core in 1 second, paper presented at the 5th ACM on International Conference on Multimedia Retrieval, June 2015. DOI: https:// doi.org/10.1145/2671188.2749398

Zhenying, H, Uehara, K, \& Shirahama, K 2018 Kobe University and Kindai University at TRECVID 2018 AVS Task. National Institute of Standards and Technology. Available at: https://www-nlpir.nist.gov/projects/tvpubs/ tv18.posters/kobe_kindai.pdf [Accessed 23 December 2020]. 
TO CITE THIS ARTICLE:

Tohline, M. 2021. A Supercut of Supercuts: Aesthetics, Histories, Databases. Open Screens, 4(1): 8, pp. 1-16. DOI: https://doi. org/10.16995/os.45

Submitted: 12 August 2020 Accepted: 03 December 2020 Published: 07 June 2021

COPYRIGHT:

(c) 2021 The Author(s). This is an open-access article distributed under the terms of the Creative Commons Attribution 4.0 International License (CC-BY 4.0), which permits unrestricted use, distribution, and reproduction in any medium, provided the original author and source are credited. See http://creativecommons.org/licenses/by/4.0/.

Open Screens is a peer-reviewed open access journal published by Open Library of Humanities. 